\title{
Development of a Korean Version of the Perceived Deficits Questionnaire-Depression for Patients with Major Depressive Disorder
}

\author{
Jae-Min Kim', Jin-Pyo Hong ${ }^{2}$, Sang-Dae Kim¹, Hee-Ju Kang ${ }^{1}$, Yong-Sung Lee ${ }^{1}$ \\ ${ }^{1}$ Department of Psychiatry, Chonnam National University Medical School, Gwangju, ${ }^{2}$ Department of Psychiatry, Sungkyunkwan University \\ School of Medicine, Seoul, Korea
}

\begin{abstract}
Objective: Cognitive symptoms are an important component of depression and the Perceived Deficits Questionnaire-Depression is one of only a few instruments available for the subjective assessment of cognitive dysfunction in depression. Thus, the present study aimed to validate a Korean version of the PDQ-D (K-PDQ-D) using patients with major depressive disorder (MDD). Methods: This study included 128 MDD patients who were assessed at study entry and 86 of these patients were then completed 12 weeks of antidepressant monotherapy. All subjects were assessed with the K-PDQ-D, the Montgomery-Asberg Depression Rating Scale (MADRS), the Sheehan Disability Scale (SDS), the EuroQol-5 dimensions questionnaire (EQ-5D), and the number of sick leave days taken in the previous week. The internal consistency, Guttman's split-half and test-retest reliabilities, factorial analyses, and concurrent and predictive validities of the K-PDQ-D were investigated.

Results: The K-PDQ-D exhibited excellent internal consistency and reliabilities, and was composed of four factors with high coefficients of determination. The concurrent validity analyses revealed that the K-PDQ-D scores were significantly correlated with the MADRS, SDS, and EQ-5D scores and the number of sick leave days taken. The K-PDQ-D scores at study entry significantly predicted changes in sick leave days and EQ-5D score from study entry to the 12-week endpoint.

Conclusion: The newly developed $K-P D Q-D$ is a reliable and valid instrument for the evaluation of subjective cognitive symptoms in MDD patients. The K-PDQ-D may assist in the gathering of unique information regarding subjective cognitive complaints, which is important for the comprehensive evaluation of patients with MDD.
\end{abstract}

KEY WORDS: Depression; Cognitive symptom; Perceived Deficits Questionnaire-Depression; Reliability; Validity.

\section{INTRODUCTION}

Depression is a common mental disorder and one of the leading causes of morbidity and disability worldwide. ${ }^{1,2)}$ Additionally, depression is associated with significant economic burdens due to missed work and/or reduced productivity at work. ${ }^{3)}$ Difficulties in attention/concentration are integrated in the diagnostic criteria of major depressive disorder (MDD). ${ }^{4)}$ Residual cognitive dysfunction has been associated with the non-remission or increased probability of relapse of a depressive episode ${ }^{5)}$ and impaired levels of functioning and work ability. ${ }^{6)}$ Thus, the improvement of cognitive function is an important aim for the treatment of depression ${ }^{7)}$ and the accu-

\footnotetext{
Received: April 8, 2015 / Revised: April 26, 2015

Accepted: May 19, 2015

Address for correspondence: Jae-Min Kim, MD, PhD

Department of Psychiatry, Chonnam National University Medical

School, 160 Baekseo-ro, Dong-gu, Gwangju 61469, Korea

Tel: +82-62-220-6143, Fax: +82-62-225-2351

E-mail:jmkim@chonnam.ac.kr
}

rate evaluation of cognitive deficits is the first step towards this goal.

Cognitive symptoms can be assessed using objective neuropsychological tests performed by clinicians or based on the subjective reports of patients. A wide variety of neuropsychological tests have been validated and used in depression research ${ }^{8)}$ but many of these tests are limited when used in clinical settings because they are too time-intensive for a busy real world practice, an expert is required to administer the test, and their associations with other functional outcomes have yet to be determined. ${ }^{9)}$ The use of subjective cognitive measures can mitigate the limitations associated with objective neuropsychological measures because the subjective tests assess the cognitive symptoms related to the individual perceptions and experiences of patients in their daily lives.

To date, no measures have been specifically designed for the evaluation of subjective cognitive symptoms in depressive patients. The Perceived Deficits Questionnaire (PDQ), which is a subjective cognitive measure, was orig-

(a) This is an Open-Access article distributed under the terms of the Creative Commons Attribution Non-Commercial License (http://creativecommons.org/licenses/by-nc/4.0) which permits unrestricted non-commercial use, distribution, and reproduction in any medium, provided the original work is properly cited. 
inally developed for use in patients with multiple sclerosis. ${ }^{10)}$ The PDQ scores of patients with multiple sclerosis are correlated with scores on depression scales but not with neuropsychological test scores, ${ }^{11)}$ which suggests that the PDQ may be used to evaluate cognitive difficulties related to depression. Hence, the PDQ has been adapted to depressive patients recently. The adapted version included minor modification compared to the original one and is called PDQ-Depression (PDQ-D). PDQ-D has begun to be validated in depressive patients. ${ }^{12-14)}$

The primary goal of the present study was to standardize a formal Korean version of the PDQ-D (K-PDQ-D). For this purpose, the internal consistency, reliability, factorial analyses, concurrent validity, and predictive validity of the K-PDQ-D were examined.

\section{METHODS}

\section{Study Participants}

The present study recruited 128 MDD patients from two university hospitals (Chonnam National University Hospital and Asan Medical Center) in South Korea from October 2013 to December 2014. The inclusion criteria for participation in this study were as follows: i) a current or new diagnosis of a major depressive episode according to the Diagnostic and Statistical Manual of Mental Disorders 4th edition, text revision (DSM-IV-TR) and confirmed with the Mini-International Neuropsychiatric Interview (MINI), ${ }^{15)}$ ii) status as an outpatient of either sex aged 19-65 years, and iii) starting an antidepressant monotherapy either as newly initiated or switched from a previous monotherapy. The exclusion criteria were as follows: i) a diagnosis of schizophrenia or other psychotic disorder, bipolar disorder, substance dependence, dementia or other neurodegenerative disease that could significantly impact cognitive functioning, or a mood disorder due to a general medical condition or substance use, ii) pregnant or breastfeeding women, or women who were 6 months post-partum, iii) the inability to read or understand the information sheet, informed consent form, or patient-report questionnaires, iv) concurrent participation in a clinical trial, and/or v) acute suicidality based on the judgment of a clinician. A total of 128 patients were evaluated at study entry and after a 12-week regimen of antidepressant monotherapy, and the remaining 86 patients completed a follow-up assessment. The present study was approved by the institutional review boards of Chonnam National University Hospital and Asan Medical Center and all participants provided written informed consent.

\section{Perceived Deficits Questionnaire}

The PDQ-D consists of 20 items that construct four domains of cognitive function: attention/concentration (Items 1, 5, 9, 13, and 17), retrospective memory (Items 2, $6,10,14$, and 18), prospective memory (Items $3,7,11,15$, and 19), and organization/planning (Items 4, 8, 12, 16, and 20). The PDQ-D was translated into Korean with minor relevant modifications that reflected the unique Korean culture and situation; the K-PDQ-D is available in the online supplement material of the present study. The administration and scoring methods for the K-PDQ-D are the same as the original PDQ. Briefly, the respondents rate the frequency of each complaint on a 5-point Likert scale anchored from never (0) to almost always (4) in a self-report form; each domain is scored out of 20 and higher scores indicate more severe cognitive dysfunction. The present study used the total score and the four domain scores for the analyses.

\section{Demographic Characteristics}

The demographic characteristics of the patients were evaluated at study entry by a clinical research coordinator using a structured case report form (CRF). Data were obtained regarding age, gender, duration of formal education, and current occupation (current employed status or not).

\section{Clinical Characteristics and Assessment Scales}

The MDD diagnoses were made by the treating clinicians using DSM-IV-TR criteria and the guidance of MINI. At study entry, the clinical research coordinators assessed all other clinical characteristics, including a history of previous depressive episodes (recurrent or first episode), using the structured CRF. Information regarding concurrent chronic medical disorders was gathered using a questionnaire that inquired about six disorders, and these data were recoded as the presence or absence of any medical disorder for the present analyses. Depression severity was measured with the Montgomery-Asberg Depression Rating Scale (MADRS), which consists of 10 items that provide a total score ranging from $0-60 .{ }^{16)}$ Disability was assessed using the Sheehan Disability Scale (SDS), which consists of three items that provide a total score ranging from $0-30 .{ }^{17)}$ Quality of life was measured using the EuroQol-5 dimensions questionnaire (EQ-5D) health status thermometer, which ranges from $0-100 .{ }^{18)}$ Sick leave days (missed school or work) in the previous week due to the depressive symptoms were also estimated. Higher scores on the MADRS and SDS, lower scores on the 
EQ-5D, and a greater number of sick leave days were considered to indicate a more severe symptomatology or impact of MDD. These assessment scales were evaluated at study entry and the 12-week endpoint of the study.

\section{Treatment-related Characteristics}

The choice of an antidepressant for each patient was determined by the independent decision of their respective clinicians. The antidepressant types were divided into three categories: i) selective serotonin reuptake inhibitors including citalopram, escitalopram, fluoxetine, paroxetine, and sertraline, ii) newer antidepressants such as bupropion, venlafaxine, and mirtazapine, and iii) older antidepressants such as amitriptyline, clomipramine, imipramine, milnacipran, nortriptyline, tianeptine, and trazodone. The use of concomitant medications such as anxiolytics and/or hypnotics was allowable if the medication was not another type of antidepressant or an antipsychotic. All adverse events reported by the patients during the study period were recorded by the clinical research coordinators.

\section{Statistical Analyses}

The descriptive data obtained at the study entry and 12-week evaluations are presented as means \pm standard deviations or as percentages, where appropriate. The scores at study entry for the patients that did and did not complete the follow-up evaluation were compared using $t$-tests or Mann-Whitney $U$-tests, respectively. The reliability analyses of the K-PDQ-D were conducted by evaluating internal consistency using Cronbach's alpha coefficient, estimating split-half reliability using Guttman's split-half coefficient, and determining test-retest reliability between the scores at study entry and follow-up scores using intraclass correlation coefficients (ICCs). For the factor analyses, a principal component analysis with varimax rotation and Kaiser normalization was used to extract the main factors, which had eigenvalues $>1.0$. The concurrent validities of the K-PDQ-D scores at study entry with the other assessment scales and the predictive validities of the K-PDQ-D scores at study entry with the changed scores on the other assessment scales (follow-up scores minus scores at study entry) were examined using Spearman's rho correlation tests. All statistical analyses were per-

Table 1. Sample characteristics

\begin{tabular}{|c|c|c|c|}
\hline \multirow{2}{*}{ Characteristic } & \multirow{2}{*}{$\begin{array}{l}\text { Sample at study entry } \\
\qquad(n=128)\end{array}$} & \multicolumn{2}{|c|}{ Followed-up sample $(n=86)$} \\
\hline & & At study entry & At follow-up \\
\hline \multicolumn{4}{|l|}{ Demographic characteristic } \\
\hline Age $(y r)$ & $47.0 \pm 11.5$ & $48.8 \pm 11.1^{\star}$ & NA \\
\hline Gender (women) & $93(72.7)$ & $60(69.8)$ & NA \\
\hline Education (yr) & $11.7 \pm 4.0$ & $10.9 \pm 4.2^{+}$ & NA \\
\hline Occupation (have) & $49(38.3)$ & $32(37.2)$ & NA \\
\hline \multicolumn{4}{|l|}{ Clinical characteristic } \\
\hline Recurrent depression & $32(25.0)$ & $20(23.3)$ & NA \\
\hline Chronic medical disorder & $61(47.7)$ & $50(58.1)^{\dagger}$ & NA \\
\hline MADRS (score) & $28.0 \pm 7.1$ & $28.6 \pm 7.2$ & $10.0 \pm 8.7$ \\
\hline SDS (score) & $20.0 \pm 7.9$ & $20.3 \pm 8.0$ & $9.4 \pm 8.6$ \\
\hline Sick leave (days in the last week) & $2.5 \pm 2.8$ & $2.6 \pm 2.9$ & $2.0 \pm 2.5$ \\
\hline EQ-5D health status (score) & $49.7 \pm 20.1$ & $50.2 \pm 18.4$ & $66.2 \pm 21.9$ \\
\hline \multicolumn{4}{|l|}{ PDQ-K (score) } \\
\hline Total & $24.4 \pm 16.0$ & $23.3 \pm 15.5$ & $15.2 \pm 15.4$ \\
\hline Attention/concentration & $7.3 \pm 4.7$ & $6.9 \pm 4.6$ & $4.0 \pm 4.5$ \\
\hline Retrospective memory & $4.6 \pm 4.2$ & $4.5 \pm 4.1$ & $3.2 \pm 4.0$ \\
\hline Prospective memory & $5.2 \pm 4.0$ & $4.9 \pm 3.9$ & $3.9 \pm 3.9$ \\
\hline Organization/planning & $7.4 \pm 5.2$ & $7.1 \pm 5.1$ & $4.1 \pm 4.6$ \\
\hline \multicolumn{4}{|l|}{ Treatment related characteristic } \\
\hline \multicolumn{4}{|l|}{ Administered antidepressants type } \\
\hline SSRIS & $77(60.2)$ & $48(55.8)$ & NA \\
\hline Newer antidepressants & $48(37.5)$ & $36(41.9)$ & NA \\
\hline Older antidepressants & $3(2.3)$ & $2(2.3)$ & NA \\
\hline Concomitant anxiolytics/hypnotics & $71(55.5)$ & $51(59.3)$ & NA \\
\hline Reported drug side effects & $18(14.0)$ & $13(15.1)$ & NA \\
\hline
\end{tabular}

Values are presented as mean \pm standard devation or number (\%).

${ }^{*} p<0.05,{ }^{+} p<0.01$ in comparisons between those lost and follow-up by t-tests, $\chi^{2}$ tests, or Mann-Whitney U-tests, as appropriate. MADRS, Montgomery Asberg Depression Rating Scale; SDS, Sheehan Disability Scale; EQ-5D, EuroQol-5 dimensions questionnaire; K-PDQ-D, Korean version of Perceived Deficits Questionnaire-Depression; SSRIs, selective serotonin reuptake inhibitors; NA, not available. 
formed using the PASW Statistics ver. 18.0 software (IBM Co., Armonk, NY, USA).

\section{RESULTS}

\section{Patient Sample and Characteristics}

A total of $128 \mathrm{MDD}$ patients were enrolled at study entry, and $86(67 \%)$ patients followed up at 12-week end point. The demographic, clinical, and treatment-related characteristics of the 128 at study entry and 86 follow-up patients are described in the first and second columns of Table 1, respectively. Compared to the patients who did not complete the follow-up assessment, the follow-up patients were significantly older, less educated, and had more chronic medical disorders. The scores on the assessment scales, including the K-PDQ-D, did not significantly differ between these two groups (all $p>0.05$ ). The scores on the assessment scales measured at follow-up are described in the third column of Table 1; in general, the assessment scale scores improved after 12 weeks of antidepressant monotherapy treatment (all $p<0.01$ ).

\section{Reliability Analyses}

The reliability analyses of the K-PDQ-D are summarized in Table 2. In the sample at study entry, the Cronbach's alpha and Guttman's split-half coefficients were high for the total scores as well as for each of the four domain scores on the K-PDQ-D. In the follow-up sample, the ICCs between the study entry and follow-up scores on the K-PDQ-D were high for the total scores and for all four domains. Overall, the K-PDQ-D exhibited good results in all reliability analyses.

\section{Factor Structure of the PDQ-K}

The factorial analyses of the K-PDQ-D in the sample at study entry are summarized in Table 3. Four factors with eigenvalues $>1.0$ were extracted and together they explained $62.8 \%$ of the variance. The four-factor structure of the K-PDQ-D was similar to the original PDQ and 16 of the 20 K-PDQ-D items (80\%) exactly matched the factor structure of the original PDQ (bold type in Table 3).

\section{Concurrent Validity}

The concurrent validities of the K-PDQ-D scores at study entry with the other assessment scales in the sample at study entry are summarized in Table 4 . The higher total scores and domain scores on the K-PDQ-D were significantly correlated with higher scores on the MADRS, the number of sick leave days, and the lower scores on the EQ-5D health status. The higher total scores and attention/concentration and organization/planning domain scores were significantly correlated with higher SDS scores. Overall, these data suggest that subjectively worse cognitive function was cross-sectionally associated with a

Table 3. Factor structure of the Korean version of Perceived Deficits Questionnaire-Depression (K-PDQ-D) at study entry $(n=128)$

\begin{tabular}{|c|c|c|c|c|}
\hline $\begin{array}{c}\text { PDQ-K } \\
\text { item number }\end{array}$ & Factor 1 & Factor 2 & Factor 3 & Factor 4 \\
\hline 1 & & & & 0.411 \\
\hline 2 & & & 0.712 & \\
\hline 3 & & & 0.801 & \\
\hline 4 & & 0.776 & & \\
\hline 5 & & & & 0.724 \\
\hline 6 & & & 0.633 & \\
\hline 7 & 0.611 & & & \\
\hline 8 & & 0.735 & & \\
\hline 9 & & 0.652 & & \\
\hline 10 & & & 0.583 & \\
\hline 11 & 0.444 & & & \\
\hline 12 & & 0.717 & & \\
\hline 13 & & & & 0.431 \\
\hline 14 & 0.604 & & & \\
\hline 15 & 0.543 & & & \\
\hline 16 & & 0.423 & & \\
\hline 17 & 0.756 & & & \\
\hline 18 & & & 0.410 & \\
\hline 19 & 0.545 & & & \\
\hline 20 & & 0.481 & & \\
\hline$\%$ variance & 18.1 & 18.0 & 15.4 & 11.3 \\
\hline Eigenvalue & 3.6 & 3.6 & 3.1 & 2.3 \\
\hline
\end{tabular}

Table 2. Results of reliability analyses of the Korean version of Perceived Deficits Questionnaire-Depression (K-PDQ-D)

\begin{tabular}{|c|c|c|c|}
\hline \multirow{2}{*}{ K-PDQ-D } & \multicolumn{2}{|c|}{ Sample at study entry $(n=128)$} & \multirow{2}{*}{$\frac{\text { Followed-up sample }(\mathrm{n}=86)}{\text { Intraclass correlation coefficients }}$} \\
\hline & Cronbach's alpha coefficients & Guttman's split half coefficients & \\
\hline Total & 0.928 & 0.947 & 0.598 \\
\hline Attention/concentration & 0.751 & 0.725 & 0.501 \\
\hline Retrospective memory & 0.827 & 0.805 & 0.622 \\
\hline Prospective memory & 0.750 & 0.720 & 0.671 \\
\hline Organization/planning & 0.847 & 0.836 & 0.438 \\
\hline
\end{tabular}


Table 4. Concurrent validity of the Korean version of Perceived Deficits Questionnaire-Depression (K-PDQ-D) with other assessment scales at study entry $(n=128)$

\begin{tabular}{|c|c|c|c|c|}
\hline K-PDQ-D & MADRS & SDS & Sick leave days & EQ-5D health status \\
\hline Total & $+0.394^{\ddagger}$ & $+0.304^{\ddagger}$ & $+0.443^{\ddagger}$ & $-0.313^{\ddagger}$ \\
\hline Attention/concentration & $+0.403^{\ddagger}$ & $+0.350^{\ddagger}$ & $+0.376^{\neq}$ & $-0.310^{\ddagger}$ \\
\hline Retrospective memory & $+0.249^{\dagger}$ & +0.166 & $+0.330^{\ddagger}$ & $-0.265^{\dagger}$ \\
\hline Prospective memory & $+0.293^{\dagger}$ & +0.166 & $+0.425^{\ddagger}$ & $-0.236^{\dagger}$ \\
\hline Organization/planning & $+0.395^{\ddagger}$ & $+0.320^{\ddagger}$ & $+0.378^{\ddagger}$ & $-0.270^{\dagger}$ \\
\hline
\end{tabular}

Values are Spearman's rho coefficients

$p<0.01,{ }^{\dagger} p<0.001$.

MADRS, Montgomery Asberg Depression Rating Scale; SDS, Sheehan Disability Scale; EQ-5D, EuroQol-5 dimensions questionnaire.

Table 5. Predictive validity of the Korean version of Perceived Deficits Questionnaire-Depression (K-PDQ-D) measured at study entry with changes in other assessment scales scores from the study entry to follow-up $(n=86)$

\begin{tabular}{|c|c|c|c|c|}
\hline K-PDQ-D & MADRS & SDS & Sick leave days & EQ-5D health status \\
\hline Total & -0.014 & -0.030 & +0.190 & -0.196 \\
\hline Attention/concentration & -0.049 & -0.083 & $+0.213^{*}$ & -0.163 \\
\hline Retrospective memory & +0.029 & -0.014 & $+0.217^{*}$ & $-0.235^{\star}$ \\
\hline Prospective memory & +0.055 & +0.037 & +0.012 & $-0.277^{\dagger}$ \\
\hline Organization/planning & -0.050 & -0.031 & +0.200 & -0.092 \\
\hline
\end{tabular}

Values are Spearman's rho coefficients.

${ }^{*} p<0.05,+p<0.01$.

MADRS, Montgomery Asberg Depression Rating Scale; SDS, Sheehan Disability Scale; EQ-5D, EuroQol-5 dimensions questionnaire.

decrease in all symptoms and functions related to depression.

\section{Predictive Validity}

The predictive validities of the K-PDQ-D scores at study entry with the changed scores on the other assessment scales from the study entry to follow-up are summarized in Table 5. The higher attention/concentration domain scores on the K-PDQ-D were significantly correlated with increase in the number of sick leave days, and the higher retrospective memory domain scores were significantly correlated with an increase in the number of sick leave days and lower EQ-5D health status scores. Additionally, higher prospective memory was significantly correlated with lower EQ-5D health status stores.

\section{DISCUSSION}

The principal findings of the present instrument standardization study using Korean MDD patients were that the newly developed K-PDQ-D showed good internal consistency and split-half and test-retest reliabilities and that it had a similar factor structure to the original PDQ version with high coefficients of determination. Furthermore, the K-PDQ-D reflected various symptoms and functions that were cross-sectionally related to depression and predicted the number of sick leave days taken from work and the health-related quality of life after 12 weeks of antidepressant treatment.

Despite the high prevalence and negative impact of cognitive dysfunction, the validities of subjective instruments for the evaluation of the cognitive function of depressive patients have only recently begun to be investigated. ${ }^{12-14)}$ The present study is one of in this area and it demonstrated significant findings. The coefficients on the Cronbach's alpha and Guttman's split-half analyses were high for the total scores and all four domain scores, which indicates that the questionnaire construction of the K-PDQ-D was consistent across the 20 items. The test-retest reliability at study entry and after 12 weeks of treatment was also good, which suggests that the K-PDQ-D reflected particular individual characteristics, even after treatment.

A study of multiple sclerosis patients found the original PDQ to be composed of four factors ${ }^{10)}$ and a recent study of whiplash disorder patients also extracted four factors. ${ }^{19)}$ The two PDQ studies that focused on depressive patients did not conduct factorial analyses ${ }^{12,13)}$ and, therefore, the present study was the first to investigate the factor structure of the PDQ using depressive patients. Interestingly, despite the use of different study populations, the same four main factors were extracted from the K-PDQ-D in the present study as in the original version of the PDQ. ${ }^{10)}$ 
Furthermore, there was a substantial overlap (80\%) in the item composition of each factor. These findings suggest that the PDQ can be used to assess patients with depression as well as those with physical disorders and that PDQ scores can be compared in similar contexts among different disease groups.

The K-PDQ-D scores were significantly correlated with the MADRS and SDS scores as well as the number of sick leave days and EQ-5D health status. These are encouraging findings which indicate that subjective cognitive dysfunction as measured by the K-PDQ-D reflected not only depressive symptoms per se but also disability, absenteeism, and quality of life, which are multi-dimensional aspects of depression. The prospective data of the present study demonstrated that some of the domain scores on the K-PDQ-D significantly predicted sick leave days taken from work and health-related quality of life but not the severity of depression or disability after 12 weeks of antidepressant monotherapy. Objective assessments of cognitive performance have been shown to significantly predict later impaired work ability in depressive patients ${ }^{6}$ but the present findings regarding subjective cognitive dysfunction are novel in this field of research. These findings suggest that the K-PDQ-D may have unique or different psychometric properties relative to other assessment scales of depression and, therefore, it would be a rational to administer the K-PDQ-D during depression research and clinical practice to predict work productivity and quality of life. However, as with other novel findings, these results should be reexamined using a larger depression cohort as well as using other patient populations with physical disorders.

The strengths of the present study include the use of a structured research protocol, well-recognized and standardized scales, and a prospective study design, which is rare in instrument standardization studies. The limitations of the present study include the fact that the sociodemographic data, clinical characteristics, and burden of MDD were unknown for the excluded patient populations due to the particular study inclusion and exclusion criteria. The particular treatment modalities for each patient were determined by the respective treating clinicians rather than by any formal guidelines and, therefore, inter-clinician variability may have affected the observed outcomes. The sample size was not sufficient for the new scale development, particularly for the factorial analyses. Additionally, the sample was drawn from two university hospitals, and therefore did not represent the Korean depressive patients as a whole. However, the demographic and clinical char- acteristics of the patients in the present study were comparable to those in a representative Korean clinical study of depression that included over 700 patients (mean age: 47.9 years, $74.4 \%$ female, and $34.2 \%$ with chronic medical disorders). ${ }^{20)}$

In conclusion, the present study demonstrated that the K-PDQ-D was a reliable and valid instrument for the assessment of depressive patients. Recently, cognitive symptoms have begun to be considered as one of the primary outcomes of depression treatment ${ }^{21)}$ and the efficacies of several antidepressants for the improvement of cognitive function in MDD patients have been investigated. $^{22,23)}$ The K-PDQ-D, which is a brief questionnaire for the evaluation of subjective cognitive symptoms in depressive patients, could offer a standard and efficient approach to facilitate the identification of cognitive symptoms and aid the progression toward treatment goals. It is strongly recommended that this instrument be administered as a complementary tool to the existing assessment scales for the gathering of unique information that may be critical for the comprehensive evaluation of depressive patients.

\section{acknowledgments}

This study was funded by H. Lundbeck A/S. The funder had no further role in study design; in the collection, analysis and interpretation of data; in the writing of the report; or in the decision to submit the paper for publication. Prof. Jae-Min Kim has received grant from Ministry of Health and Welfare, Republic of Korea; Lundbeck; Eli Lilly; and Otsuka. Prof. Jin-Pyo Hong has received research funding from Ministry of Health and Welfare, Republic of Korea; and Lundbeck. The other authors report no conflict of interest.

\section{REFERENCES}

1. Kessler RC, Berglund P, Demler O, Jin R, Koretz D, Merikangas $\mathrm{KR}$, et al; National Comorbidity Survey Replication. The epidemiology of major depressive disorder: results from the National Comorbidity Survey Replication (NCS-R). JAMA 2003;289:3095-3105.

2. Moussavi S, Chatterji S, Verdes E, Tandon A, Patel V, Ustun B. Depression, chronic diseases, and decrements in health: results from the World Health Surveys. Lancet 2007;370:851-858.

3. Stewart WF, Ricci JA, Chee E, Hahn SR, Morganstein D. Cost of lost productive work time among US workers with depression. JAMA 2003;289:3135-3144.

4. American Psychiatric Association. Diagnostic and statistical manual of mental disorders. 4th ed. Text revision. Washington, DC:American Psychiatric Press Inc;2000.

5. Judd LL, Akiskal HS, Maser JD, Zeller PJ, Endicott J, Coryell $\mathrm{W}$, et al. Major depressive disorder: a prospective 
study of residual subthreshold depressive symptoms as predictor of rapid relapse. J Affect Disord 1998;50:97-108.

6. Jaeger J, Berns S, Uzelac S, Davis-Conway S. Neurocognitive deficits and disability in major depressive disorder. Psychiatry Res 2006;145:39-48.

7. Danner M, Hummel JM, Volz F, van Manen JG, Wiegard $\mathrm{B}$, Dintsios CM, et al. Integrating patients' views into health technology assessment: Analytic hierarchy process (AHP) as a method to elicit patient preferences. Int J Technol Assess Health Care 2011;27:369-375.

8. Greer TL, Kurian BT, Trivedi MH. Defining and measuring functional recovery from depression. CNS Drugs 2010;24: 267-284.

9. Evans VC, Iverson GL, Yatham LN, Lam RW. The relationship between neurocognitive and psychosocial functioning in major depressive disorder: a systematic review. $J$ Clin Psychiatry 2014;75:1359-1370.

10. Sullivan MJL, Edgley K, Dehoux E. A survey of multiple sclerosis, part 1: perceived cognitive problems and compensatory strategy use. Can J Rehabil 1990;4:99-105.

11. Lovera J, Bagert B, Smoot KH, Wild K, Frank R, Bogardus $\mathrm{K}$, et al. Correlations of perceived deficits questionnaire of multiple sclerosis quality of life inventory with Beck depression inventory and neuropsychological tests. $J$ Rehabil Res Dev 2006;43:73-82.

12. Lawrence C, Roy A, Harikrishnan V, Yu S, Dabbous O. Association between severity of depression and self-perceived cognitive difficulties among full-time employees. Prim Care Companion CNS Disord 2013;15. doi: 10.4088/PCC.12m01469. [Epub ahead of print]

13. Fehnel SE, Forsyth BH, Dibenedetti DB, Danchenko N, François C, Brevig T. Patient-centered assessment of cognitive symptoms of depression. CNS Spectr 2013 Sep 25:1-10. [Epub ahead of print]

14. Lam RW, Saragoussi D, Danchenko N, Rive B, Lamy FX, Brevig T. Psychometric validation of Perceived Deficits Questionnaire-Depression (PDG-D) in patients with major depressive disorder (MDD). Value Health 2013;16:A330.

15. Sheehan DV, Lecrubier Y, Sheehan KH, Amorim P, Janavs J, Weiller E, et al. The Mini-International Neuropsychiatric Interview (M.I.N.I.): the development and validation of a structured diagnostic psychiatric interview for DSM-IV and ICD-10. J Clin Psychiatry 1998;59 Suppl 20:22-33.

16. Montgomery SA, Asberg M. A new depression scale designed to be sensitive to change. Br J Psychiatry 1979;134:382-389.

17. Leon AC, Olfson M, Portera L, Farber L, Sheehan DV. Assessing psychiatric impairment in primary care with the Sheehan Disability Scale. Int J Psychiatry Med 1997;27: 93-105.

18. EuroQol Group. EuroQol--a new facility for the measurement of health-related quality of life. Health Policy 1990; 16:199-208.

19. Takasaki H, Chien CW, Johnston V, Treleaven J, Jull G. Validity and reliability of the perceived deficit questionnaire to assess cognitive symptoms in people with chronic whiplashassociated disorders. Arch Phys Med Rehabil 2012;93: 1774-1781.

20. Kim JM, Kim SW, Stewart R, Kim SY, Yoon JS, Jung SW, et al. Predictors of 12-week remission in a nationwide cohort of people with depressive disorders: the CRESCEND study. Hum Psychopharmacol 2011;26:41-50.

21. McIntyre RS. Using measurement strategies to identify and monitor residual symptoms. J Clin Psychiatry 2013;74 Suppl 2:14-18.

22. Raskin J, Wiltse CG, Siegal A, Sheikh J, Xu J, Dinkel JJ, et al. Efficacy of duloxetine on cognition, depression, and pain in elderly patients with major depressive disorder: an 8-week, double-blind, placebo-controlled trial. Am J Psychiatry 2007;164:900-909.

23. McIntyre RS, Lophaven S, Olsen CK. A randomized, double-blind, placebo-controlled study of vortioxetine on cognitive function in depressed adults. Int $J$ Neuropsychopharmacol 2014;17:1557-1567. 